\title{
Neutralitätspflicht für (Partei-)Amtsträger? Verfassungsrechtliche Grenzen der Einflussnahme bei Durchführung eines Mitgliedervotums
}

\author{
Alexander Hobusch
}

Anfang des vergangenen Jahres gab es kaum ein Thema, das so engagiert diskutiert wurde, wie das anstehende Mitgliedervotum der SPD. Die nahezu 460.000 Parteimitglieder waren aufgerufen, darüber zu befinden, ob die Parteiführung den mit der CDU/CSU ausgehandelten Koalitionsvertrag abschließen sollte. Hierbei stellen sich nicht nur politische, sondern auch rechtliche Fragen, etwa ob der Parteivorstand, der (möglicherweise) eigene Interessen bei Mitgliedervoten verfolgt, Beschränkungen unterworfen ist hinsichtlich der Einwirkung auf die Mitglieder, sei es etwa durch Zuschriften, Veröffentlichungen oder sonstige Einflussversuche.

Gerade die Gegner einer möglichen Großen Koalition - und damit der Linie der Parteiführung - fühlten sich in der Diskussion nicht hinreichend berücksichtigt. Vielmehr wurde dem Parteivorstand vorgeworfen, unfair zu spielen und die Mitglieder vor dem Votum unzulässig zu beeinflussen und nur einseitig in seinem Sinne zu informieren. ${ }^{1}$ Beispielsweise wurde deutliche Kritik daran laut, dass den Briefwahlunterlagen, die jedes Mitglied nach Hause geschickt bekam, ein dreiseitiges Begleitschreiben des Verhandlungsteams beilag, das die Erfolge der Verhandlungen lobte und mit allen Unterschriften der Parteiführung beziehungsweise der Verhandlungsteilnehmer endete. ${ }^{2}$ Außerdem wurde die einseitige Berichterstattung in der Parteizeitschrift vorwärts kritisiert. Das Sonderheft des vorwärts begann ebenfalls mit einer zweiseitigen Einführung durch die Verhandlungsteilnehmer, in der die Ergebnisse positiv beurteilt wurden. ${ }^{3}$ Auch die hohe Frequenz an Zusendungen via E-Mail, in denen die Erfolge der Verhandlungen herausgestellt wurden, wurde moniert.

Die besondere Bedeutung dieses Mitgliedervotums und die zunehmende Nutzung dieses Instruments legen nahe zu untersuchen, ob dem Parteivorstand bei der Durchführung rechtliche Grenzen für die Einwirkung auf die Mitglieder gesetzt sind. Die Grundfrage ist, ob die Verfassung oder das einfache Gesetz hier eine Beschränkung auferlegen und damit eine Zurückhaltungspflicht beziehungsweise Neutralitätspflicht seitens des Vorstandes zu beachten gilt. Konkret ist zu prüfen, ob die Anforderungen im Fall des SPD-Votums eingehalten worden sind.

1 Siehe etwa Jusos in der SPD Düsseldorf, Erklärung zum Mitgliedervotum, http://www.jusosduesseldorf.de/wp-content/uploads/2018/02/erklaerung_zur_groko_jusos_duesseldorf.pdf (Abruf am 15. Januar 2019).

2 Vgl. Adrian Arab, Ärger über einseitige Begleitschreiben der Parteispitze, in: Die Welt online vom 20. Februar 2018, https://www.welt.de/politik/deutschland/article173771729/SPD-Mitgliederentscheid-Aerger-ueber-einseitige-Begleitschreiben-des-Vorstandes.html (Abruf am 15. Januar 2019); Max Holscher / Christian Teevs, Ja oder nein, Genossen?, in: Spiegel online vom 3. März 2018, http://www.spiegel.de/politik/deutschland/spd-mitgliedervotum-groko-oder-nicht-tag-derentscheidung-a-1196131.html (Abruf am 15. Januar 2019).

3 Vgl. „Vorwort“, in: Vorwärts, Ausgabe 01/02 2018, S. 1 f., https://www.vorwaerts.de/content/ vorwaerts-ausgabe-januarfebruar-2018 (Abruf am 27. Januar 2019). 


\section{Innerparteiliche Demokratie und demokratische Grundsätze}

Das Grundgesetz schreibt den Parteien in Art. 21 I 3 unmissverständlich die innerparteiliche Demokratie vor. Ihre innere Ordnung muss „demokratischen Grundsätzen“ entsprechen.

\subsection{Zweck des Gebotes innerparteilicher Demokratie}

Der Zweck der innerparteilichen Demokratie lässt sich zunächst, auch historisch, negativ bestimmen: Die Verpflichtung der Parteien, ihre Organisation und ihre Verfahren demokratisch zu gestalten, soll verhindern, dass undemokratische Parteien existieren und am Willensbildungsprozess teilnehmen. ${ }^{4}$ Dies zielt nicht nur auf Parteien mit „Führerprinzip“, sondern ist insgesamt auch auf die Bekämpfung oligarchischer Tendenzen in den Parteien gerichtet. ${ }^{5}$ Positiv formuliert machen demokratische Strukturen die Mitwirkung des einzelnen Bürgers in der Partei erst möglich. ${ }^{6}$ Gerade Parteien, die die politische Willensbildung prägen ${ }^{7}$ und kanalisieren, müssen selbst demokratisch organisiert sein. ${ }^{8}$ Es wäre ein Widerspruch, wenn Parteien bei ihrer Mitwirkungsaufgabe selbst nicht an demokratische Grundsätze gebunden wären”, schließlich sind sie die „berufenen Vorkammern“ unserer Demokratie. ${ }^{10}$ Die Chancen des Bürgers, sich am demokratischen Prozess überhaupt zu beteiligen, hängen damit maßgeblich von den tatsächlichen wie rechtlichen Ausgestaltungen der Parteiorganisation ab. ${ }^{11}$ Nach diesen Möglichkeiten der Einflussnahme bemisst sich auch der „Wirklichkeitsgehalt" der Volkssouveränität. ${ }^{12}$ Innerparteiliche Demokratie sichert also die Offenheit des politischen Prozesses ${ }^{13}$ und zwar an seiner Quelle ${ }^{14}$.

4 Vgl. Hans-Peter Schneider, Die Institution der politischen Partei in der Bundesrepublik Deutschland, in: Dimitris Th. Tsatsos / Dian Schefold / Hans-Peter Schneider (Hrsg.), Parteienrecht im europäischen Vergleich - Die Parteien in den demokratischen Ordnungen der Europäischen Gemeinschaft, Baden-Baden 1990, S. 151 - 218, S. 195.

5 Vgl. Karl-Heinz Seifert, Die politischen Parteien im Recht der Bundesrepublik Deutschland, Köln 1975, S. 190.

6 Vgl. Martin Morlok, in: Horst Dreier (Hrsg.), Grundgesetz Kommentar, Band II, Tübingen 2015, Art. 21 Rn. 123.

7 Vgl. Dimitris Th. Tsatsos, Ein Recht auf innerparteiliche Opposition, in: Rudolf Bernhardt / Wilhelm Karl Geck / Günther Jaenicke / Helmut Steinberger (Hrsg.), Völkerrecht als Rechtsordnung, Berlin / Heidelberg / New York 1983, S. 997 - 1032, S. 998, spricht von der faktischen Priorität der Parteien bei der Willensbildung; siehe zur hervorragenden Bedeutung der Parteien in der modernen parlamentarischen Demokratie auch BVerfGE 44, 125, 145.

8 Vgl. Karl-Heinz Seifert, a.a.O. (Fn. 5), S. 189.

9 Vgl. Jörn Ipsen, in: Michael Sachs (Hrsg.), Grundgesetz Kommentar, München 2018, Art. 21 Rn. 53; Klaus Seidel, Direkte Demokratie in der innerparteilichen Willensbildung, Frankfurt am Main 1998, S. 33.

10 Vgl. Dieter Grimm, in: Ernst Benda / Werner Maihofer / Hans-Jochen Vogel (Hrsg.), Handbuch des Verfassungsrechts der Bundesrepublik Deutschland, Band I, Berlin / New York 1995, \$ 14 Rn. 36.

11 Vgl. Dimitris Th. Tsatsos, a.a.O. (Fn. 7), S. 998.

12 Vgl. ebenda.

13 Vgl. Dittmar Hahn, Innerparteiliche Demokratie, Köln 1973, S. 93 ff.

14 Vgl. Konrad Hesse, Grundzüge des Verfassungsrechts der Bundesrepublik Deutschland, Heidelberg 1999, Rn. 175. Siehe auch Martin Morlok, a.a.O. (Fn. 6), Art. 21 Rn. 123; Dimitris Th. 


\subsection{Gehalt der demokratischen Grundsätze}

Dem Textbefund folgend fällt auf, dass das Grundgesetz in Art. 21 I 3 nicht auf das Demokratieprinzip an sich verweist. Es fordert demnach nicht etwa, dass die Parteistruktur „demokratisch“ sein muss, sondern vielmehr nur, dass die Ausgestaltung „demokratischen Grundsätzen“ entsprechen muss. Was darunter im Einzelnen zu verstehen ist, welchen Gehalt diese Normierung im Grundgesetz also hat, ist seit Jahren lebhaft diskutiert worden.

Zunächst ist festzustellen, dass die „demokratischen Grundsätze“ bereits dem Wortlaut nach verwandt sind mit Artikel 20 I und Artikel 28 I GG, die ebenfalls von demokratischen Grundsätzen sprechen. ${ }^{15}$ Teilweise wird die demokratische Binnenstruktur der Parteien auch aus dem demokratischen Prinzip abgeleitet. ${ }^{16}$ Eine lückenlose Übertragung des Demokratieprinzips auf die innerparteilichen Grundsätze ist dabei aber wohl nicht möglich ${ }^{17}$ und auch nicht sachgerecht ${ }^{18}$, widerspräche auch dem Textbefund, der eben nur von den Grundsätzen handelt. ${ }^{19}$ Die Unterschiede zwischen Staat und Partei sind bei der Betrachtung der innerparteilichen Demokratie zu beachten, wenn ein staatsrechtliches Prinzip auf eine nichtstaatliche Einrichtung angewendet werden soll. ${ }^{20}$ Bei einer Übernahme von Elementen aus dem demokratischen Prinzip müssen diese also ,vereinstypisch“ ${ }^{21}$ betrachtet werden. ${ }^{22}$ Bei den demokratischen Grundsätzen kann es schon aus der Terminologie heraus nur um Mindeststandards ${ }^{23}$ gehen, nicht hingegen um ein wünschenswertes demokratisches Maximum. ${ }^{24}$ In Rechnung zu stellen ist auch, dass es sich bei Parteien um „Freiwilligenorganisationen mit Tendenzcharakter" ${ }^{25}$ handelt, so dass die Parteien zur Abgrenzung im Wettbewerb mit Konkurrenten ein gewisses Maß an Durchsetzungskraft und Geschlossenheit benötigen. ${ }^{26}$ Im Spannungsfeld zwischen Gestaltungsfreiheit und innerparteilicher Demokratie ${ }^{27}$ verbleibt den Parteien also ein Spielraum. ${ }^{28}$

Tsatsos, Ein Recht auf innerparteiliche Opposition, in: ders. / Martin Morlok / Konrad Hesse / Dimitris Stefanou (Hrsg.), Verfassung - Parteien - Europa - Abhandlungen aus den Jahren 19621998, Baden-Baden 1998, S. 423 - 466, S. 442; vgl. Klaus Seidel, a.a.O. (Fn. 9), S. 33.

15 Vgl. Hans-Peter Schneider, a.a.O. (Fn. 4), S. 195 f.; Klaus Seidel, a.a.O. (Fn. 9), S. 75, 89.

16 Vgl. Karl-Heinz Seifert, a.a.O. (Fn. 5), S. 189; Vgl. Martin Morlok, a.a.O. (Fn. 6), Art. 21 Rn. 123.

17 Vgl. Martin Morlok, a.a.O. (Fn. 6), Art. 21 Rn. 125.

18 Vgl. Jörn Ipsen, a.a.O. (Fn. 9), Art. 21 Rn. 54; Dimitris Th. Tsatsos, a.a.O. (Fn. 14), S. 440; Martin Morlok / Thilo Streit, Mitgliederentscheid und Mitgliederbefragung - Rechtsprobleme direkter Demokratie in den politischen Parteien, in: ZRP, 29. Jg. (1996), H. 11, S. 447 - 455, S. 449; Georg König, Die Verfassungsbindung der politischen Parteien, Berlin 1993, S. 37.

19 Vgl. Dimitris Th. Tsatsos, a.a.O. (Fn. 14), S. 444.

20 Vgl. Wilhelm Henke, Das Recht der politischen Parteien, Göttingen 1972, S. 49; ders., in: Wolfgang Kahl / Christian Waldhoff I Christian Walter (Hrsg.), Bonner Kommentar zum Grundgesetz, Heidelberg 2014, Art. 21 Rn. 262; Klaus Stern, Das Staatsrecht der Bundesrepublik Deutschland, Band I, München 1984, S. 444 f.; vgl. Klaus Seidel, a.a.O. (Fn. 9), S. 33, S. 73.

21 Karl-Heinz Seifert, a.a.O. (Fn. 5), S. 190.

22 Vgl. Dimitris Th. Tsatsos, a.a.O. (Fn. 14), S. 444 spricht von der Berücksichtigung der Parteifunktionen.

23 Vgl. Martin Morlok / Thilo Streit, a.a.O. (Fn. 18), S. 449.

24 Vgl. Jörn Ipsen, a.a.O. (Fn. 9), Art. 21 Rn. 55; Karl-Heinz Seifert, a.a.O. (Fn. 5), S. 191.

25 Martin Morlok, a.a.O. (Fn. 6), Art. 21 Rn. 125.

26 Vgl. ebenda.

27 Vgl. dazu auch Klaus Seidel, a.a.O. (Fn. 9), S. 90.

28 Vgl. Martin Morlok, Parteiengesetz, Baden-Baden 2013, Vorb. $\$ 6$ Rn. 2; ders. I Thilo Streit, a.a.O. (Fn. 15), S. 449. 
Als demokratische Grundsätze lassen sich daher die folgenden ausmachen: Parteiämter dürfen nur aufgrund von Wahlen vergeben werden, die Legitimation muss also von der Mitgliederversammlung abhängen. ${ }^{29}$ Die Willensbildung muss mit anderen Worten „von unten nach oben" verlaufen. ${ }^{30}$ Entscheidendes Element innerparteilicher Demokratie sind gerade periodische Wahlen, die die Mitbestimmung des Einzelnen zu sichern vermögen. ${ }^{31}$ Dazu gehören auch die Freiheit und Gleichheit sowie die Geheimheit der Wahlen, also ebenso dem Grunde nach die Wahlrechtsgrundsätze aus Art. 38 I 1 GG mit Ausnahme der Unmittelbarkeit. ${ }^{32} \mathrm{Zu}$ den demokratischen Grundsätzen zählt auch das Mehrheitsprinzip, wobei der Schutz der Minderheit notwendige Ergänzung des Mehrheitsprinzips ist. ${ }^{33}$ Die Minderheit der Gegenwart muss die Chance haben, die Mehrheit der Zukunft zu werden; es muss folglich die Möglichkeit eines „Machtwechsels“ geben, und die Minderheit muss sich ausreichend artikulieren können. ${ }^{34}$ Dies setzt eine angemessene Entscheidungs- und Willensfreiheit der Mitglieder, Antrags- sowie Rederechte und den Schutz vor Missbrauch der Verbandsgewalt voraus. ${ }^{35}$ Nicht das Mehrheitsprinzip für sich genommen macht eine Entscheidung demokratisch, sondern ein geregelter Kampf um die Mehrheit. ${ }^{36}$ Somit lässt sich aus dem Mehrheitsprinzip und dem damit verbundenen Minderheitsschutz auch ein Recht auf innerparteiliche Opposition ableiten ${ }^{37}$; schließlich ist das Recht zur Bildung einer Opposition eines der wesentlichen Elemente der freiheitlich demokratischen Ordnung ${ }^{38}$, ein „demokratisches Minimum“39 und damit auch Bestandteil der innerparteilichen Demokratie. ${ }^{40}$ Zum Schutz der Minderheit und ihrer Möglichkeit, einmal Mehrheit zu werden, lässt sich durchaus von der bei Parteien und Bürgern verwendeten Terminologie der Chancengleichheit sprechen, hier also von innerparteilicher Chancengleichheit. ${ }^{41}$ Dies

29 Vgl. etwa Klaus Stern, a.a.O. (Fn. 20), S. 445 f.; Karl-Heinz Seifert, a.a.O. (Fn. 5), S. 191.

30 BVerfGE 2, 40; vgl. Klaus Stern, a.a.O. (Fn. 20), S. 445; Karl-Heinz Seifert, a.a.O. (Fn. 5), S. 191; Dittmar Hahn, a.a.O. (Fn. 13), S. 52; Martin Morlok / Thilo Streit, a.a.O. (Fn. 18), S. 449.

31 Vgl. Jörn Ipsen, a.a.O. (Fn. 9), Art. 21 Rn. 59; Karl-Heinz Seifert, a.a.O. (Fn. 5), S. 191; Klaus Stern, a.a.O. (Fn. 20), S. 446.

32 Vgl. Christoph Gusy, in: Ekkehart Stein / Ehrhard Denninger / Wolfgang Hofmann-Riem (Hrsg.), Alternativ-Kommentar zum Grundgesetz für die Bundesrepublik Deutschland (AK-GG), Art. 21 Rn. 70; Wilhelm Henke, a.a.O. (Fn. 20), Art. 21 Rn. 288; Rudolf Streinz, in: Hermann von Mangoldt / Friedrich Klein / Christian Starck, Grundgesetz Kommentar, Band 2, München 2018, Art. 21 Rn. 168 mit weiteren Nachweisen; Klaus Seidel, a.a.O. (Fn. 9), S. 104.

$33 \mathrm{Zu}$ allem Vorgenannten vgl. Jörn Ipsen, a.a.O. (Fn. 9), Art. 21 Rn. 59.

34 Vgl. ebenda; Hans-Peter Schneider, a.a.O. (Fn. 4), S. 195. Das BVerfG fordert auch gleiche Chancen im freien und offenen Prozess der Meinungs- und Willensbildung des Volkes (BVerfGE 44, 125, 149), erstreckt diese Gleichheit aber auch auf das Vorfeld politischer Willensbildung (so bereits BVerfGE 8, 51, 68).

35 Vgl. Karl-Heinz Seifert, a.a.O. (Fn. 5), S. 191.

36 Vgl. Friedrich Grawert, Parteiausschluß und innerparteiliche Demokratie, Heidelberg 1987, S. 63.

37 Vgl. Dimitris Th. Tsatsos / Martin Morlok, Parteienrecht, Heidelberg 1982, S. 63 ff.; Hans-Peter Schneider, a.a.O. (Fn. 4), S. 195; Dimitris Th. Tsatsos, a.a.O. (Fn. 14), S. 464; Martin Morlok, in: Peter Häberle I ders. / Vassilios Skouris (Hrsg.), Festschrift für Dimitris Th. Tsatsos, Baden-Baden 2003, S. 408 - 447, S. 430. Zum Begriff etwa Dimitris Th. Tsatsos, a.a.O. (Fn. 7), S. 1002 f.

38 Vgl. Dimitris Th. Tsatsos, a.a.O. (Fn. 7), S. 1002.

39 Ders., a.a.O. (Fn. 14), S. 445.

40 Vgl. Hans-Peter Schneider, a.a.O. (Fn. 4), S. 200.

41 Vgl. Karl-Heinz Seifert, a.a.O. (Fn. 5), S. 267 f.; Martin Morlok / Thilo Streit, a.a.O. (Fn. 18), S. 449, 451, sprechen von einem „offenen, chancengleichen Wettbewerb“, der gewährleistet sein muss; vgl. Christoph Gusy, a.a.O. (Fn. 32), Art. 21 Rn. 77. 
ist konsequent, denn die Chancengleichheit selbst wird auch nicht nur in Art. 21 GG, sondern vielmehr ebenso vornehmlich im demokratischen Prinzip verortet. ${ }^{42}$

Die Möglichkeit, ein Mitgliedervotum oder eine sonstige basisdemokratische Urabstimmung durchzuführen, gehört dagegen nicht zu den Minimalanforderungen an die innerparteiliche Demokratie, sondern stellt eine darüber hinausgehende Option der Parteien zur Strukturierung der innerparteilichen Willensbildung dar. ${ }^{43}$ Dies bedeutet allerdings nicht, dass die Ausgestaltung eines Mitgliedervotums weniger strengen Regeln unterläge: Die Grundsätze innerparteilicher Demokratie gelten für den Willensbildungsprozess in den Parteien unabhängig davon, ob dieser nun vornehmlich in basisdemokratischen oder repräsentativen Verfahren stattfindet.

Zu klären ist in diesem Kontext die Frage des Adressaten: Wenn von Chancengleichheit die Rede ist, dann ist davon zumeist das Verhältnis der Parteien oder des Bürgers zum Staat gemeint. Eine Erweiterung auf Private als Adressat der politischen Chancengleichheit wird gemeinhin abgelehnt, die Gewährleistung richtet sich vielmehr gegen die staatliche Gewalt. ${ }^{44}$ Allerdings liefe die Gewährleistung der innerparteilichen Demokratie und damit das Individualrecht auf Chancengleichheit ${ }^{45}$ beziehungsweise der chancengleichen Mitwirkung des Einzelnen ${ }^{46}$ leer, wenn man Parteien als Adressat der Chancengleichheit von vornherein ausnehmen würde. Insofern ist anerkannt, dass die Chancengleichheit des Einzelnen über demokratische Grundsätze Teil der Gewährleistung des Art. 21 I 3 GG ist, der unmittelbar gegen die Parteien wirkt. ${ }^{47}$

\subsection{Chancengleiche Willensbildung auch in den Parteien}

Chancengleichheit in diesem Sinne bedeutet die rechtliche Absicherung des demokratischen Prozesses in der Partei, also eine ungestörte und ungefilterte Willensbildung von unten nach oben und damit einhergehend eine Beschränkung der Oligarchisierungstendenz. ${ }^{48}$ Die Binnenorganisation der Partei und auch die Handlungen ihrer Akteure müssen somit dergestalt sein, dass das einzelne Mitglied gleiche Chancen auf Teilhabe am Meinungsbildungsprozess hat. Die innerparteiliche Demokratie ist selbst eine Wettbewerbsordnung,

42 Vgl. Andreas Kißlinger, Das Recht auf politische Chancengleichheit, Baden-Baden 1998, S. 56; siehe auch die Auswertung der Rechtsprechung auf S. 27 ff. mit weiteren Nachweisen.

43 Dazu mit weiteren Nachweisen Klaus Seidel, a.a.O. (Fn. 9), S. 91.

44 Vgl. Andreas Kißlinger, a.a.O. (Fn. 42), S. 147; Hanns-Rudolf Lipphardt, Die Gleichheit der politischen Parteien vor der öffentlichen Gewalt, Berlin 1975, 119 ff.; Christian Jülich, Chancengleichheit der Parteien, Berlin 1967, 98 ff.; Hubert Meyer, Kommunales Parteien- und Fraktionenrecht, Baden-Baden 1990, S. 63; Karl-Heinz Seifert, a.a.O. (Fn. 5), S. 135 ff.; weitergehend Wilhelm Henke, a.a.O. (Fn. 20), S. 251.

45 Vgl. Dimitris Th. Tsatsos, a.a.O. (Fn. 14), S. 448; Martin Morlok, a.a.O. (Fn. 37), S. 430; Wilhelm Henke, a.a.O. (Fn. 20), Art. 21 Rn. 320, spricht von „Bürgergleichheit“.

46 Vgl. Martin Morlok, a.a.O. (Fn. 6), Art. 21 Rn. 124 ff.; ders., a.a.O. (Fn. 28), Vorb. $\$ 6$ Rn. 3; Uwe Volkmann, in: Karl-Heinrich Friauf / Wolfram Höfling (Hrsg.), Berliner Kommentar zum Grundgesetz, Berlin 2000, Art. 21 Rn. 42; Andreas Kißlinger, a.a.O. (Fn. 42), S. 87 ff.

47 Vgl. Andreas Kißlinger, a.a.O. (Fn. 42), S. 148; Georg König, a.a.O. (Fn. 18), S. 31 ff.; Dimitris Th. Tsatsos / Martin Morlok, a.a.O. (Fn. 37), S. 53 f.; Martin Morlok, a.a.O. (Fn. 37), S. 430; ders., a.a.O. (Fn. 28), Vorb. $\$ 6$ Rn. 3.

48 Vgl. Martin Morlok, a.a.O. (Fn. 37), S. 429. 
denn Demokratie bedeutet auch hier Kampf verschiedener Ansichten um den richtigen Weg. ${ }^{49}$ Die Regeln des fairen Wettbewerbs der Meinungen müssen festgelegt und befolgt werden. ${ }^{50}$ Im Einzelnen sind hier beispielhaft ein Recht auf Teilnahme an Veranstaltungen, an Wahlen und Abstimmungen, aber auch Informationsrechten zu nennen. ${ }^{51}$

\section{Einfachgesetzliche Ausgestaltung}

Was das Prinzip innerparteilicher Demokratie und den damit verbundenen Anspruch auf gleichberechtigte Teilhabe am politischen Prozess innerhalb der Parteien angeht, sind die Regelungen im Parteiengesetz als Konkretisierung der demokratischen Grundsätze denkbar knapp und nicht abschließend. ${ }^{52}$ Wesentliches Merkmal demokratischer Entscheidung ist laut Parteiengesetz vor allem das Prinzip der Mehrheit, das in $\$ 15$ PartG seinen Niederschlag findet. Dass diesem aber kraft Verfassung ein Minderheitsschutz immanent ist, wurde bereits oben gezeigt. Jedenfalls im einfachen Recht findet sich derlei Schutz allerdings nur sehr schwach ausgeprägt wieder; vielmehr handelt es sich um eine Sicherung von Mindestelementen. ${ }^{53} \mathrm{Zu}$ nennen ist hier etwa $\$ 15$ III 1 PartG, der der Minderheit Antragsrechte zubilligt. ${ }^{54}$ Selbiges gilt für das Antragsrecht für Vertreter der beiden nächstniedrigeren Stufen bei Versammlungen höherer Gebietsverbände, das sich in $\$ 15$ III 2 PartG findet. ${ }^{55}$ Die Absicherung des freien Mandates in $\$ 15$ III 3 PartG schützt ebenfalls die Minderheit vor einem „Durchentscheiden“ der Mehrheit. ${ }^{56}$ Daneben haben auch Quoten zur Einberufung eines Parteitages minderheitsschützenden Charakter. ${ }^{57}$ Neben diesen genannten Beispielen hat der Gesetzgeber Minderheitsrechte zurückhaltend positiviert, denn mit dem Mehrheitsprinzip wird eher die Absicht verfolgt, den Parteien zur Geschlossenheit ${ }^{58} \mathrm{zu}$ verhelfen. ${ }^{59}$

49 Vgl. ebenda.

50 Vgl. ebenda, S. 430; Rudolf Streinz, a.a.O. (Fn. 32).

51 Vgl. Georg König, a.a.O. (Fn. 18), S. 41; Martin Morlok, a.a.O. (Fn. 37), S. 430.

52 Vgl. Sophie-Charlotte Lenski, Parteiengesetz und Recht der Kandidatenaufstellung, Baden-Baden 2011, $\$ 15$ Rn. 1; Martin Morlok, a.a.O. (Fn. 28), Vorb. \$6 Rn. 2. Anders etwa Wilhelm Henke, a.a.O. (Fn. 20), S. 53, der sehr zurückhaltend meint, dass ,im Zweifel alle demokratischen Anforderungen" durch den Gesetzgeber niedergelegt seien. Dies kann so aber nicht überzeugen, denn der Gesetzgeber kann nicht alle Elemente demokratischer Grundsätze positiv niederlegen, ebenso wenig wie der Gesetzgeber alle Elemente des Demokratieprinzips abschließend festlegen kann.

53 Vgl. Hans-Peter Schneider, a.a.O. (Fn. 4), S. 196.

54 Vgl. Friedrich Grawert, a.a.O. (Fn. 36), S. 63.

55 Vgl. Hans-Peter Schneider, a.a.O. (Fn. 4), S. 200.

56 Vgl. ebenda.

57 Vgl. ebenda.

58 Zur Rolle der Geschlossenheit für Parteien etwa Dimitris Th. Tsatsos / Martin Morlok, a.a.O. (Fn. 37), S. 66; Stefan Schieren, Parteiinterne Mitgliederbefragungen: Ausstieg aus der Professionalität? Die Beispiele der SPD auf Bundesebene und in Bremen sowie der Bundes-F.D.P., in: ZParl, 27. Jg. (1996), H. 2, S. 214 - 229, S. 225; Dimitris Th. Tsatsos, a.a.O. (Fn. 14), S. 446; kritisch zum Druck auf die Mitglieder schon etwa Hans See, Volkspartei im Klassenstaat oder das Dilemma der innerparteilichen Demokratie, Reinbek bei Hamburg 1972, S. 38 f.; weitere Nachweise bei Dimitris Th. Tsatsos, a.a.O. (Fn. 7), S. 1000.

59 Vgl. Hans-Peter Schneider, a.a.O. (Fn. 4), S. 200. 


\section{Mitgliedervotum}

\subsection{Zweck des Votums ${ }^{60}$}

Die basisdemokratische Entscheidung einer Sach- oder Personalfrage ist die wohl reinste Anwendung demokratischer Grundsätze. Hier besteht die Möglichkeit, dass alle Mitglieder unmittelbar an einer Entscheidung mitwirken können. Im Vergleich zu dem ansonsten verfolgten mittelbaren beziehungsweise repräsentativen Aufbau der Parteien ist dies eine wesentliche Erweiterung der Rechte des einzelnen Mitglieds. Mitgliedervoten haben nachweislich Mobilisierungscharakter. ${ }^{61}$ Sie erreichen, dass sich mehr Parteimitglieder an der innerparteilichen Willensbildung beteiligen, und schöpfen daraus auch ein Mehr an Legitimation. Die gesteigerte demokratische Legitimation eines Basisentscheides beflügelt auch die integrative Funktion eines Mitgliederentscheides. ${ }^{62}$ Wenn alle Mitglieder zu einem Problem befragt worden sind, ist es auch für die unterlegene Seite einfacher, sich mit dem ihr unliebsamen Ergebnis anzufreunden. Man spricht in der Folge davon, dass es bei einem Mitgliedervotum keine Verlierer gibt. ${ }^{63}$ Gleichzeitig führt ein Basisvotum grundsätzlich dazu, dass oligarchische Tendenzen bekämpft werden, was ebenso als positiver Nebeneffekt direktdemokratischer Elemente gelten kann. ${ }^{64}$ Daneben sind „Partei-Plebiszite“ jedenfalls grundsätzlich gut für die interne Diskussion und eine innerparteiliche Opposition ${ }^{65}$. Durch die direkte Partizipation des Mitgliedes werden auch die ansonsten bestehenden Kommunikationswege durchbrochen, es findet also ein Kommunikationsfluss zum einfachen Mitglied statt, wie es sonst nicht üblich ist. ${ }^{66}$ Neben diesen grundlegenden Erwägungen liegt aber genau hier eine Gefahr: Zwar wird der Kommunikationsfluss zum einfachen Mitglied

60 Die grundsätzliche Zulässigkeit basisdemokratischer Entscheide soll hier nicht näher thematisiert werden, dies ist mittlerweile hinlänglich diskutiert worden; vgl. Bernd Grzeszick / Jochen Rauber, in: Hans Hofmann / Hans-Günter Henneke (Hrsg.), Grundgesetz, Köln 2018, Art. 21 Rn. 110 mit weiteren Nachweisen; kritisch trotz allem etwa Martin Pagenkopf, in: ZRP 2018, S. 37 ff., der auf „Sonderrechte" der Mitglieder im Vergleich zum Wahlvolk abstellt und gleichzeitig das freie Mandat verletzt sieht (wohl eher durch Koalitionsverträge an sich), aber dabei außer Acht lässt, dass auch eine Entscheidung eines Parteivorstandes durch dessen vorangegangene Wahl durch die Mitglieder legitimiert ist. Auch hier hätten folglich die Parteimitglieder „Sonderrechte“. Diese ergeben sich zudem zwanglos aus der Mitgliedschaft in der Partei und sind mitnichten verfassungsrechtlich bedenklich, schließlich ist es unschädlich, dass die direkte Mitarbeit in politischen Parteien, die die Willensbildung in der Parteiendemokratie maßgeblich prägen, zu einem Mehr an Partizipationschancen führt. Gleichzeitig wird - wenig schlüssig - den sonst zur Entscheidung berufenen Delegierten ein Mehr an „Erfahrungswissen“ attestiert und festgestellt, das einzelne Parteimitglied könne doch „ohne sachverständige Kommentierung und Erläuterung“ die Koalitionsvereinbarung gar nicht verstehen, was schlechterdings nicht nachvollziehbar erscheint. Zum SPD-Mitgliedervotum 2013 siehe in concreto BVerfG, in: DVBl 2014, S. $3 \mathrm{ff}$.

61 Vgl. Stefan Schieren, a.a.O. (Fn. 58), S. 222.

62 Vgl. Bernd Becker, Wozu denn überhaupt Parteimitglieder? Zum Für und Wider verstärkter parteiinterner Partizipationsmöglichkeiten. Eine Antwort auf den Beitrag von Stefan Schieren in Heft 2 / 1996 der ZParl, in: ZParl, 27. Jg. (1996), H. 4, S. 712 - 718, S. 718.

63 Vgl. Stefan Schieren, a.a.O. (Fn. 58), S. 223.

64 Vgl. Dittmar Hahn, a.a.O. (Fn. 13), S. 64.

65 Vgl. Joachim Raschke, Innerparteiliche Opposition, Hamburg 1974, S. 127; einschränkend ders., Organisierter Konflikt in westeuropäischen Parteien, Wiesbaden 1977, S. 144, der darauf hinweist, dass Mitgliedervoten auch zu einer Spaltung der innerparteilichen Opposition führen können.

66 Joachim Raschke, a.a.O. (Fn. 65), S. 144. 
verstärkt, die Diskussion wird angeregt, weil die Entscheidung durch alle Mitglieder gleichermaßen getroffen wird. Starke Beeinflussung der Basis durch Parteieliten kann aber die grundsätzlichen Vorteile einer direktdemokratischen Abstimmung umkehren: Fällt eine Entscheidung zwar durch die Abstimmung aller Mitglieder, allerdings unter deutlicher Beeinflussung, kann dies die befriedende Wirkung einer Abstimmung relativieren. Auch basisdemokratische Entscheidungen sind also nicht (allein) dazu in der Lage, oligarchische Tendenzen zu neutralisieren; sie leisten eher einen Beitrag zur Etablierung einer innerparteilichen Opposition und sind diskursfördernd.

\subsection{Anforderungen aus dem Grundsatz innerparteilicher Demokratie}

Auf die Frage nach den normativen Anforderungen für Mitgliederentscheide, welche Maßstäbe also an ihre Verankerung in den Satzungen der Parteien anzulegen sind, soll hier nicht weiter eingegangen werden; dies ist ebenso hinreichend diskutiert wie die grundsätzliche Zulässigkeit basisdemokratischer Entscheide. ${ }^{67}$ Hier kommt es nicht auf die satzungsmäßige Fixierung an, sondern es geht um das tatsächliche Handeln des Parteivorstandes, wodurch die Chancengleichheit möglicherweise verletzt wurde.

\section{(1) Parallelen zur Öffentlichkeitsarbeit der Regierung im Wahlkampf?}

Abstrahiert man den Sachverhalt und versetzt sich in die Lage des einzelnen Mitglieds, das sich im Vorfeld des Votums einer Informationskampagne des Parteivorstandes ausgesetzt sieht, der die Abstimmung in seinem Sinne beeinflussen möchte, so ist dies jedenfalls auf den ersten Blick vergleichbar mit der Regierung, die vor Wahlen durch Öffentlichkeitsarbeit Einfluss auf die Entscheidung der Wähler nehmen will.

Aus den Grundsätzen der Freiheit der Wahl, der Chancengleichheit der Parteien und des Demokratieprinzips ist zum Schutze des demokratischen Wettbewerbs in der Judikatur des Bundesverfassungsgerichts die so genannte Neutralitätspflicht für Amtsträger beziehungsweise Staatsorgane entwickelt worden. Diese soll sicherstellen, dass die Wähler ihre Wahlentscheidung in einem freien, offenen Prozess der Willensbildung fällen können ${ }^{68}$, also weder der Staat noch Private Druck ausüben dürfen. Weil die Willensbildung vom Volk zum Staat verlaufen soll und nicht umgekehrt ${ }^{69}$, ist es staatlichen Stellen nicht erlaubt, in amtlicher Funktion auf die Willensbildung des Volkes bei Wahlen und in deren Vorfeld ${ }^{70}$ einzuwirken. ${ }^{71}$ Ergreifen Staatsorgane als solche Partei zugunsten oder zuungunsten einer Partei oder eines Wahlbewerbers, ist die Chancengleichheit verletzt ${ }^{72}$, was wegen der dadurch erschütterten demokratischen Legitimation auf die Gültigkeit von Wahlen durchschlagen kann. ${ }^{73}$

67 Martin Morlok / Thilo Streit, a.a.O. (Fn. 18), S. $451 \mathrm{ff}$.

68 Vgl. BVerfGE 20, 56, 97; 44, 125, 139; zuletzt BVerfG, Urteil vom 16. Dezember 2014, - 2 BvE 2/14 - „Schwesig“, Rn. 28.

$69 \mathrm{Vgl}$. BVerfGE 44, 125, 140.

70 Zur Anwendbarkeit außerhalb von Wahlkampfzeiten siehe BVerfGE 148, 11, 25.

71 Vgl. BVerfGE 138, 102, 111; 148, 11, 25.

72 So bereits BVerfGE 44, 125, 146; zuletzt E 148, 11, 25.

73 Vgl. BVerfGE 44, 125, 154. 
Die an sich zulässige Öffentlichkeitsarbeit findet dort ihre Grenze, wo die Wahlwerbung beginnt ${ }^{74}$; staatliche Öffentlichkeitsarbeit darf also nicht genutzt werden, um Werbung für einzelne im Wettbewerb stehende Parteien zu machen. ${ }^{75}$ Nimmt ein Amtsinhaber also am politischen Meinungskampf teil, was dem Grunde nach möglich sein muss ${ }^{76}$, so muss ein Rückgriff auf die mit dem Amt verbundenen „Mittel und Möglichkeiten“ unterbleiben: Werden durch Amtsträger die Autorität des Amtes oder die mit dem Amt zusammenhängenden Ressourcen ,in spezifischer Weise“ genutzt, unterliegt der Amtsträger der Neutralitätspflicht. ${ }^{77}$ Die Abgrenzung zwischen dem Handeln als „Politiker“ oder „Amtsträger“ ist von den Umständen des jeweiligen Einzelfalles abhängig. ${ }^{78}$ Gerade in der Vorwahlzeit verdichtet sich die Neutralitätspflicht zu einem „Gebot äußerster Zurückhaltung“ und zum „Verbot" der Veröffentlichung sogenannter Arbeits-, Leistungs-, oder Erfolgsberichte aus Haushaltsmitteln. ${ }^{79}$ In der Vorwahlzeit kann also auch schon die sachliche Information eine unzulässige Wahlwerbung darstellen. ${ }^{80}$ Die „heiße Wahlkampfphase“, die damit besonderem Schutz unterliegt, ist dabei nicht genau zu bestimmen; als Orientierungspunkt soll jedenfalls der Zeitpunkt gelten, an dem der Wahltermin bestimmt wird (\$ 16 BWahlG). ${ }^{81}$

Ob diese Grundsätze auf die innerparteiliche Willensbildung übertragbar sind, ist fraglich. Wie oben dargestellt, sind die Chancengleichheit und auch die Freiheit der Wahl sehr wohl Elemente der innerparteilichen Demokratie, damit erscheint eine Verletzung aus denselben Gründen möglich, denn schließlich beeinflusst der Vorstand einseitig die Meinungsbildung im Verband. Allerdings muss bei der Anwendung innerhalb der Parteien beachtet werden, dass es sich bei diesen zwar um Organisationen zwischen Gesellschaft und Staat, aber eben nicht um staatliche Akteure ${ }^{82}$ handelt. Auch handelt es sich weder um „amtliche Autorität" noch um staatliche Gelder, die eingesetzt werden. Anders als der Staat wirken Parteien auch nach außen und stehen in Konkurrenz zu anderen Parteien. Aber auch hier muss die Freiheit und Gleichheit der Wahl gewährleistet werden, denn die Willensbildung des Volkes soll vor allem durch die Parteien ermöglicht werden. Diese Willensbildung setzt die Parteien nämlich voraus ${ }^{83}$, sie sind als Mittler zwischen Bürgern und Staatsorganen dazu „berufen, die Aktivbürger (...) mit dem Ziel der Beteiligung an der Willensbildung (...) zusammenzufassen" 84 .

Die Grundsätze, die hier die Chancengleichheit der Wahlbewerber und der Parteien in Bezug auf staatliche Akteure gewährleisten sollen, sind wegen oben genannter Bedenken zwar nicht unverändert übertragbar, allerdings ihrem Gedanken nach durchaus auf die Parteien anzuwenden. Dafür bedarf es aber eines eigenen, parteienspezifischen Maßstabs.

74 Vgl. BVerfGE 44, 125, $148 \mathrm{ff}$.

75 Vgl. BVerfGE 44, 125, 148 ff; zuletzt etwa E 148, 11, 28 f.

76 Vgl. BVerfGE 44, 125, 141.

77 BVerfGE 44, 125, Leitsatz (2).

78 Vgl. BVerfGE 138, 102, 117 f. auch zu weiteren Kriterien und Fallgruppen; siehe auch E 148, $11,31 \mathrm{ff}$.

79 Dazu BVerfGE 44, 125, 126 (LS 8)

80 Vgl. BVerfGE 44, 125, $151 \mathrm{f}$.

81 Vgl. BVerfGE 44, 125, 153.

82 Zur Stellung der Parteien etwa: Martin Morlok, a.a.O. (Fn. 6), Art. 21 Rn. 22.

83 So bereits BVerfGE 44, 125, 145.

84 BVerfGE 44, 125, 145. 
(2) Parteienspezifischer Maßstab: Bedeutung chancengleicher Mitwirkung für den politischen Prozess

Es sprechen gute Gründe dafür, auch im innerparteilichen Bereich - wenn auch abgeschwächt - eine Zurückhaltungspflicht anzunehmen. Einerseits handelt es sich zwar um keine staatlichen Wahlen, andererseits sind Parteien die Bindeglieder zwischen Gesellschaft und Staat und damit der staatlichen Sphäre eben nicht komplett entzogen. Die Willensbildung der Parteien ist nicht ohne Grund kraft Verfassung demokratischen Grundsätzen unterworfen: Sie ist für ein Funktionieren der Parteiendemokratie schlechterdings notwendige Voraussetzung. Insofern ist es zwar richtig, dass den Parteien bei der konkreten Ausgestaltung der innerparteilichen Demokratie Freiräume zu belassen sind; dies bedeutet aber keinen Verzicht auf wesentliche Elemente eines fairen Meinungsprozesses, und um ein solches handelt es sich bei dem Grundsatz chancengleicher Betätigung des Einzelnen. Denkt man die Parteiendemokratie vom Individuum, also vom einzelnen Parteimitglied her, so muss gerade hier der Willensbildungsprozess an der Quelle geschützt werden. Den Parteien kommt nicht ohne Grund eine „Zwischenstellung“ zu, sie sollen doch gerade die Verbindung zu den Staatsorganen fördern. Sind Parteien aber hier in einer faktischen Monopolstellung, muss die Chancengleichheit des Bürgereinflusses ${ }^{85}$ auch innerhalb der Parteien Anwendung finden. Sind sie die einzig realistische Möglichkeit des Einzelnen, an der politischen Willensbildung teilzunehmen und das Vehikel des Bürgers, um seine Ansichten und Standpunkte in den parlamentarischen Prozess einzuspeisen, muss gerade dieser Mechanismus geschützt sein. Zudem soll nach der vielbeschworenen Formel die Willensbildung in den Parteien doch gerade von „unten nach oben“ verlaufen, nicht umgekehrt. Setzte man einer Beeinflussung durch den Parteivorstand gar keine Grenzen, würde dies den Prozess möglicherweise umkehren. Zwar verbliebe die letztendliche Entscheidung bei den Mitgliedern, aber diese könnten durch den Vorstand „auf Linie“ gebracht werden. Auch bei staatlichen Wahlen ist dies kein Argument; hier genügt auch eine mögliche Beeinflussung der Wahlentscheidung. Die ohnehin bestehende Gefahr der Oligarchisierung ${ }^{86}$ in den Parteien würde noch zunehmen, wenn Vorstände und damit die Eliten der Partei auf ihr gesamtes Arsenal an Mitteln unbegrenzt zurückgreifen könnten, um ihre eigenen Ansichten dem „Parteivolk“ zu oktroyieren. Im Detail sind diese Möglichkeiten sehr wohl beachtlich, denn die Führung hat etwa die Kontrolle über die Parteibürokratie, die selbst keinen eigenen Machtapparat darstellt, sondern Instrument in den Händen der Parteiführung ist. ${ }^{87}$ Dies ist ein handfester Vorteil der Vorstände, und trägt tendenziell eher zu deren Machterhalt bei $^{88}$ - nicht zuletzt, weil die Parteibürokratie der Führung den Zugriff auf den innerparteilichen Kommunikationsprozess sichert ${ }^{89}$, der der innerparteilichen Opposition dagegen verwehrt bleibt. ${ }^{90}$ Daneben ist auch die Parteipresse, sofern sie in deutschen Parteien noch

$85 \mathrm{Zu}$ den Unterschieden und Parallelen zur Chancengleichheit der Parteien etwa Hans Herbert von Arnim, Der strenge und der formale Gleichheitssatz, in: DÖV 1984, S. 85 - 92, S. 89; dazu auch BVerfGE 8, $51 \mathrm{ff}$.

86 Vgl. Martin Morlok, a.a.O. (Fn. 6), Art. 21 Rn. 123; Bernd Becker, a.a.O. (Fn. 62), S. 713 beide mit weiteren Nachweisen.

87 Vgl. Joachim Raschke, a.a.O. (Fn. 65), S. 114; ders., a.a.O. (Fn. 66), S. 138.

88 Vgl. Bodo Zeuner, Innerparteiliche Demokratie, Berlin 1970, S. 103.

89 Vgl. Joachim Raschke, a.a.O. (Fn. 66), S. 139.

90 Vgl. ebenda. 
vorhanden ist ${ }^{91}$, ein Machtmittel der Parteiführung, mit dem innerparteiliche Opposition bekämpft werden kann. ${ }^{92}$ So bestehen viele Möglichkeiten der Führung, den innerparteilichen Willensbildungsprozess zu beeinflussen.

(3) Parteienspezifischer Maßstab: Parteienfreiheit als korrelierendes Recht

Auf der anderen Seite ist zu betrachten, dass es sich bei Parteien um „Tendenzbetriebe“93 handelt. Die Tendenzfreiheit als Ausfluss der inneren Parteienfreiheit ermöglicht es einer Partei, alle internen Strukturen auf die inhaltliche Programmatik der Partei zu verpflichten. ${ }^{94}$ Der Vorstand ist notwendiges Organ der Partei ( $\$ 8$ I 1 PartG). Er soll die Partei nach außen vertreten und die laufenden Geschäfte führen ( $\$ 11$ III PartG). ${ }^{95}$ Dabei ist der Vorstand als Exekutivorgan vor allem für das ,politische Tagesgeschäft “ zuständig, während die Mitgliederversammlung die grundlegenden Entscheidungen fällt. ${ }^{96}$ Für diese Repräsentationsfunktion ${ }^{97}$ muss der Vorstand auch in der Lage sein, die Mehrheitsmeinung derjenigen, die ihn legitimiert haben, nach außen zu tragen. Hier steht dem Parteivorstand umgekehrt sogar ein Monopol der Vertretung zu. ${ }^{98}$ Wäre es einem Vorstand nicht möglich, Partei zu ergreifen, dann wäre der gesamte Verband handlungsunfähig.

Allerdings lässt sich hier durchaus differenzieren: Bei der Vertretung der Partei nach außen, muss dem Vorstand im Zweifel ein weiterer Spielraum eingeräumt werden, denn diese ist Grund seiner Existenz und eine seiner wesentlichen Aufgaben ${ }^{99}$, insbesondere in rechtlicher Hinsicht. Der Vorstand macht die Partei handlungsfähig und reagiert auf aktuelle Entwicklungen in der Politik. Eine andauernde Konsultation eines Parteitages für einzelne Sachfragen ist nicht praktikabel, würde den Vorstand seiner Aufgabe berauben und die Partei ihrer Handlungsfähigkeit.

Anders liegt die Sache aber bei einer Wirkung des Vorstandes nach innen. Hier ist zu beachten, dass die Parteiführung nicht „autonom“ von den Mitgliedern entscheiden soll; vielmehr muss sie zur Partei hin offen sein, ähnlich wie der Staat zu den Parteien. ${ }^{100}$ In Bezug auf den innerparteilichen Willensbildungsprozess hat die Führung keine Repräsentationsfunktion, wie sie nach außen besteht, aber eine politische Leitungsfunktion; sie verfasst also Konzepte, arbeitet Positionen aus und ähnliches. Mit diesen Ergebnissen seiner Arbeit muss der Vorstand selbstverständlich auch nach innen wirken ${ }^{101}$, was auch Teilnahme am Diskussionsprozess bedeutet, nicht aber eine Umkehrung der Willensbildung.

91 Vgl. Hans-Peter Schneider, a.a.O. (Fn. 4), S. 193.

92 Vgl. Bodo Zeuner, a.a.O. (Fn. 88), S. 103.

93 Vgl. Martin Morlok, a.a.O. (Fn. 6), Art. 21 Rn. 60 f.

94 Vgl. ebenda, Art. 21 Rn. 61; zu dem beschriebenen Spannungsverhältnis auch mit weiteren Nachweisen Rudolf Streinz, a.a.O. (Fn. 32).

95 Vgl. Wilhelm Henke, a.a.O. (Fn. 20), Art. 21 Rn. 295; Sophie-Charlotte Lenski, a.a.O. (Fn. 52), $\$ 8$ Rn. 3.

96 Vgl. Sophie-Charlotte Lenski, a.a.O. (Fn. 52), $\$ 8$ Rn. 3.

97 Siehe dazu Wilhelm Henke, a.a.O. (Fn. 20), S. 76.

98 Vgl. Sophie-Charlotte Lenski, a.a.O. (Fn. 52), \$8 Rn. 3.

99 Vgl. Wilhelm Henke, a.a.O. (Fn. 20), S. 76.

100 Vgl. ebenda.

101 Vgl. Sophie-Charlotte Lenski, a.a.O. (Fn. 52), \$11 Rn. 23. 
(4) Parteienspezifischer Maßstab: Freiheit der Wahl

Um die innerparteiliche Willensbildung besonders im Vorfeld von Abstimmungen vor Verfälschungen zu schützen, ist neben der Chancengleichheit auch die Wahlfreiheit ins Feld zu führen, die verlangt, dass die Abstimmenden ihr Votum in einem „freien, offenen Prozess der Meinungsbildung [...] fällen können "102. Der Wähler soll vor Beeinträchtigungen seiner Entscheidungsfreiheit geschützt werden. ${ }^{103}$ Die Wahlfreiheit gilt - wie oben bereits erläutert - auch im innerparteilichen Bereich ${ }^{104}$ und umfasst die Neutralitätspflicht von Amtsträgern. Die Anwendbarkeit der Wahlfreiheit zeigt die besondere Sensibilität, die in direktem räumlich-zeitlichen Zusammenhang mit (auch innerparteilichen) Wahlakten zu beachten ist.

Das oben hergeleitete Recht des Einzelnen auf chancengleiche Mitwirkung und Teilhabe am innerparteilichen Meinungswettbewerb muss mit dem Recht der Parteien auf effektive Ausgestaltung der innerparteilichen Willensbildung und damit einem Element der Parteienfreiheit in Einklang gebracht werden. Bei einer Abwägung ist aber zu berücksichtigen, dass die Parteienfreiheit nicht unbeschränkt gilt, sondern das Grundgesetz diese ausdrücklich begrenzt ${ }^{105}$ und die Parteien auf die innerparteiliche Demokratie verpflichtet. Aus der dem Willensbildungsprozess zukommenden, für die Parteiendemokratie konstitutiven Bedeutung ${ }^{106}$ ist es von Verfassungs wegen notwendig, ihn vor Manipulation zu bewahren. Dabei müssen die Belange der Parteien und die Funktionsfähigkeit hinreichend berücksichtigt werden. Aufgrund der Freiheit der Wahl, die jedenfalls sinngemäß auch im Innerparteilichen gelten muss, folgt eine Verdichtung der Anforderungen mit zunehmender Nähe zum Abstimmungstag.

\section{(5) Parteienspezifischer Maßstab: Entscheidungsrecht des Vorstandes?}

Daran ändert auch nichts, dass der Vorstand ohne Mitgliedervotum selbst über den Koalitionsvertrag hätte entscheiden können. Denn zum einen ist beim Mitgliederentscheid gemäß $\$ 13$ IV Organisationsstatut der SPD eben auch ein anderer Initiator als der Vorstand denkbar; zum anderen könnte sich ein solches Verfahren sogar gegen Entscheidungen oder Beschlüsse des Vorstandes selbst richten ( $\$ 13$ I Organisationsstatut der SPD), womit der in diesem Beitrag gezeigte Konflikt zwischen abstimmender Basis und dem diese Basis beeinflussenden Vorstand noch deutlicher hervortreten würde. Außerdem lässt sich der Vorstand durch die Initiierung eines Mitgliedervotums auf ein Verfahren mit einer Beteiligung der Mitglieder ein und erkennt damit den Wert dieser Abstimmung an. Diesen beschrittenen Weg kann er nicht durch eine den oben hergeleiteten Grundsätzen widersprechende Beeinflussung zu seinen Gunsten abändern. Kurzum: Nur weil der Vorstand ohne Mitgliedervotum entscheiden könnte, senkt dies nicht die Anforderungen, die sich aus den Grundsätzen der innerparteilichen Demokratie für die Durchführung eines Basisentscheids ergeben.

102 Siehe etwa BVerfGE 44, 125, 139.

103 Vgl. BVerfGE 79, 161, $165 \mathrm{f}$.

104 Vgl. Georg König, a.a.O. (Fn. 18), S. 76; siehe dazu mit weiteren Nachweisen Fn. 32.

105 Vgl. Martin Morlok, a.a.O. (Fn. 6), Art. 21 Rn. 66.

106 Vgl. ebenda, Art. 21 Rn. 123; zur Notwendigkeit der Parteien zur Ermöglichung wirksamen Bürgereinflusses etwa BVerfGE 11, 266, 273, ständige Rechtsprechung; zuletzt BVerfG, Urteil vom 27. Februar 2018, - 2 BvE 1/16, Rn. 41. 
Auch ob ein solcher Entscheid nun verbindlich oder unverbindlich getroffen wird, ist für die rechtliche Bewertung in Bezug auf die chancengleiche Teilhabe daran unbeachtlich, da auch die konsultative Mitgliederbefragung eine faktische Bindungswirkung in der Partei entfaltet. ${ }^{107}$ Plakativ ausgedrückt: "Wenn der Souverän spricht, so spricht er auch verbindlich!"108

\section{(6) Konkretisierte Anforderungen}

Zwar ist im Falle eines Mitgliedervotums oftmals - aber nicht zwangsläufig ${ }^{109}$ - der Parteivorstand der Initiator, womit ihm auch nicht verwehrt sein darf, sich zur Thematik zu äußern und sich zu positionieren. ${ }^{110}$ Hier liegt der maßgebliche Unterschied zu den Fällen der staatlichen Öffentlichkeitsarbeit: In Tendenzorganisationen wie den Parteien, die nach innen wie nach außen wirken, muss es den Trägern von Parteiämtern in viel größerem Ausmaß möglich sein, selbst auch an dem politischen Meinungskampf innerhalb ihrer Partei teilzunehmen. Denn sie bekleiden kein staatliches Amt, tragen keine „Amtsautorität“ und keine staatlichen Symbole, handeln nicht als Staatsorgane, und die Amtsmittel sind keine Haushaltsmittel der öffentlichen Hand. Insofern kann eine „Neutralität“ hier keine inhaltliche Zurückhaltung bei jeglichen Äußerungen im Sinne eines dauernden Sachlichkeitsgebotes bedeuten, sondern vielmehr nur eine solche Zurückhaltung bei Nutzung spezifischer (finanzieller) Parteimittel: Erst der Rückgriff auf Parteiressourcen macht eine Einwirkung zum Problem. ${ }^{11}$ Äußert sich etwa der Parteivorsitzende in einer Tageszeitung über seine Präferenz zum Votum, ist dies unschädlich: Amtsautorität oder die Verwendung staatlicher Symbole liegen im innerparteilichen Bereich nicht vor, auf eine „Sprecherrolle“, wie sie in der Rechtsprechung konstruiert wird, kommt es hier nicht an. Insofern kann etwa die Äußerung in einer (externen) Zeitung gar keine Verletzung der parteienspezifischen Neutralität bedeuten. Erst der Rückgriff auf sachliche oder personelle Mittel der Partei erscheint problematisch.

Grundlage einer jeden demokratischen Entscheidung ist Information. Ohne Kenntnis der Entscheidungsmöglichkeiten kann ein wirklicher Willensbildungsprozess nicht stattfinden und eine echte Auswahl nicht bestehen. Die Parteiexekutive hat einen strukturellen Wissens- und Informationsvorsprung. ${ }^{12}$ Aus dem Anspruch auf chancengleiche Mitwirkung innerhalb der Partei ergibt sich auch ein Informationsanspruch gegenüber dem Parteivorstand. Dieser muss also die Entscheidung durch sachliche Erläuterungen vorbereiten und solche auch zu anderen Abstimmungsoptionen zulassen. Für diese Aufgabe ist die Verwendung von Parteimitteln selbstverständlich zulässig.

Wegen der grundsätzlichen Anwendbarkeit der Wahlrechtsgrundsätze sind auch weitere Minimalanforderungen zur Absicherung des Willensbildungsprozesses einzuhalten. Die Wahlen müssen frei und geheim ablaufen. Der Vorstand unterliegt zwar keiner grundlegenden, strikten Neutralität wie Amtsträger; werden aber spezifische (finanzielle) „Amtsmittel“

107 Vgl. Martin Morlok / Thilo Streit, a.a.O. (Fn. 18), S. 455 mit weiteren Nachweisen.

108 Ebenda.

109 Vgl. $\$ 13$ III, IV des Organisationsstatutes der SPD.

110 Vgl. Martin Morlok/ Thilo Streit, a.a.O. (Fn. 18), S. 454.

111 Vgl. ebenda, S. 454.

112 Siehe etwa Joachim Raschke, a.a.O. (Fn. 65), S. 149 ff. 
zum innerparteilichen Meinungskampf genutzt, müssen in Anlehnung an die Rechtsprechung zur Neutralität von Amtsträgern strengere Maßstäbe angelegt werden. Dies folgt aus dem notwendigen Schutz der freien und chancengleichen innerparteilichen Willensbildung, denn die Ressourcen der Partei sind darauf gerichtet, die Willensbildung zu organisieren, nicht sie in eine Richtung zu lenken. Hier erscheint es durchaus naheliegend, bei allem, was über eine reine Positionierung beziehungsweise sachliche Information seitens des Vorstandes hinausgeht und wertenden, ja werbenden Charakter hat, den Zugriff auf finanzielle Mittel der Partei zu sperren. Damit sind werbende Zusendungen des Vorstandes an alle Mitglieder unzulässig, da diese Mittel der innerparteilichen Opposition im Meinungswettbewerb nicht zur Verfügung stehen. Nutzt der Vorstand Parteiressourcen, muss er also besonders sachlich und ausgewogen informieren.

Dafür spricht auch ein Vergleich mit Personalentscheidungen durch Basisentscheid: Treten zwei Kandidaten in einer Urwahl für ein Parteiamt an, ist Werbung für einen von ihnen mit Parteiressourcen des Vorstandes undenkbar, wenn der andere dabei ausgeklammert wird. Die SPD-Statuten sehen für den Fall einer solchen Urwahl eine gemeinsame Wahlkampfkasse vor, die von den Kandidaten zu gleichen Teilen genutzt werden kann. ${ }^{113}$ Eingenommene Spenden fließen der Partei zu, die sie ebenfalls zu gleichen Teilen ausgibt. Finanziell hat man sich also für strikte Gleichheit im Wettkampf um ein Parteiamt entschieden, was zum Schutze der chancengleichen Willensbildung von unten auch notwendig erscheint. Dies muss sinngemäß ebenso für die Nutzung finanzieller Parteiressourcen für den Meinungskampf gelten, denn inhaltliche Fragen sind mit personellen untrennbar verbunden: Auch inhaltliche Positionierungen können eine Missbilligung der Vorstandsarbeit darstellen. Der Vorstand darf aber nicht durch Zugriff auf Parteikassen seine Machtbasis sichern oder ihm unangenehme Beschlüsse verhindern.

Jedenfalls der Schutz des unmittelbaren zeitlichen Vorfelds einer Abstimmung vor gleichheitswidriger Einflussnahme unter Zuhilfenahme von Parteiressourcen muss gewährleistet sein; insofern verdichten sich die Anforderungen mit zunehmender Nähe zum Abstimmungstermin. Besonders der Wahlzettel, auf dem die Stimmabgabe erfolgt, muss frei von Beeinflussung sein, um eine freie Entscheidung der Mitglieder garantieren zu können. Auch dies ergibt sich als demokratisches Minimum ohne Weiteres aus dem Grundsatz innerparteilicher Demokratie. Damit ist im direkten Umfeld der Wahlunterlagen selbst kein Raum mehr für eine Beeinflussung des Mitglieds. Zu diesem Zeitpunkt verletzt selbst sachliche Information die innerparteiliche Wahlfreiheit.

\section{Anwendung auf den Ausgangsfall}

Im vorliegenden Fall gab es zahlreiche Zuschriften an alle Parteimitglieder per Email, zudem eine Extra-Ausgabe des vorwärts, in der der Koalitionsvertrag beleuchtet und die Erfolge der Verhandlungen herausgestellt wurden. Eine Mitarbeit von Gegnern des Koalitionsvertrages war nicht vorgesehen. Die Papiere, die jedes Mitglied per Post zugeschickt bekam, enthielten neben den Abstimmungsunterlagen ein Begleitschreiben der Parteiführung.

113 SPD Parteivorstand, Verfahrensrichtlinien zur Durchführung von Mitgliederbegehren und Mitgliederentscheid nach $\$ 13$ Abs. 7 Organisationsstatut sowie Mitgliederbefragungen nach $\$ 14$ Abs. 11 Organisationsstatut, dort unter III. Nr. 6. 
Nach den oben erarbeiteten Grundsätzen waren die werbenden Zuschriften an die Parteimitglieder unzulässig, da Parteimittel für einseitige Stellungnahmen verausgabt wurden. Jedenfalls im unmittelbaren Vorfeld der Abstimmung hätte der Vorstand keine einseitigen Stellungnahmen mehr unter Rückgriff auf die Parteiressourcen verschicken dürfen.

Zumindest bei der Versendung der Abstimmungsunterlagen ist kein Raum mehr für eine werbende Beeinflussung der Mitglieder. In dem Begleitschreiben heißt es unter anderem: „Mit der SPD in Regierungsverantwortung können wir in den nächsten Jahren viel bewegen."Anschließend werden die Erfolge in den einzelnen Politikbereichen aufgefächert. Nach Informationen zur Rücksendung folgt: „Wir als Verhandlungsteam empfehlen Dir aus Überzeugung, mit JA zu stimmen. "Unter der Grußformel erscheinen die Unterschriften des damaligen SPD-Vorsitzenden Martin Schulz, der Vorsitzenden der SPD-Bundestagsfraktion Andrea Nahles, des Generalsekretärs Lars Klingbeil sowie 34 weiterer prominente Parteimitglieder, die an den Verhandlungen beteiligt waren. Bereits in der Darstellung der vielen Vorteile der Regierungsbeteiligung ist jedenfalls eine indirekte Empfehlung zu sehen, jedoch enthält das Schreiben auch ganz explizit den Aufruf der Unterzeichner zu einem gewissen Abstimmungsverhalten. Besonders fällt ins Auge, dass dieses Empfehlungsschreiben mit organisatorischen Hinweisen zur Durchführung vermengt ist, also auch diesbezüglich keine klare Trennung vorliegt.

Den oben hergeleiteten Anforderungen an eine demokratischen Grundsätzen entsprechende Basisabstimmung genügt dies nicht. In unmittelbar mit dem Abstimmungszettel verbundenen Schreiben der Parteiführung wird eindeutig für eine der Seiten des Votums geworben, womit die Wahlfreiheit nicht mehr gewährleistet ist. Die von der Partei und damit auch vom Parteivorstand zu wahrende innerparteiliche Chancengleichheit ist damit verletzt.

\section{Fazit: Verstoß gegen demokratische Grundsätze}

Auch innerhalb der Parteien können sich einzelne Mitglieder auf chancengleiche Teilhabe am Willensbildungsprozess berufen. Es gibt nicht nur ein Recht auf innerparteiliche Opposition, sondern auch ein Recht auf innerparteiliche Chancengleichheit. Dies ergibt sich aus der Verpflichtung der Parteien auf demokratische Grundsätze aus Art. 21 I 3 GG. Aus der besonderen Bedeutung der Willensbildung in den Parteien und deren Wichtigkeit für die vom Grundgesetz vorgesehenen Parteiendemokratie ist die Führung der Partei insbesondere bei Abhaltung eines Mitgliedervotums an die Wahrung der oben genannten Chancengleichheit gebunden und daher im Vorfeld der Abstimmung zu einem gewissen Maß an Zurückhaltung verpflichtet, um den innerparteilichen Willensbildungsprozess nicht unter Rückgriff auf Parteiressourcen zu verfälschen. Die Rechtsprechung zur Öffentlichkeitsarbeit der Regierung im Vorfeld von öffentlichen Wahlen ist allerdings nicht ohne Weiteres übertragbar, sondern es bedarf eines eigenen, parteienspezifischen Maßstabes. Dieser gebietet gerade mit zunehmender Nähe zum Abstimmungstag zunehmende Zurückhaltung. Jedenfalls die Versendung von Wahlunterlagen, die mit einem werbenden Begleitschreiben verbunden sind, verstößt gegen die Chancengleichheit der einzelnen Parteimitglieder, womit auch ein Verstoß gegen die demokratischen Grundsätze aus Art. 21 I 3 GG vorliegt. 